\title{
Approximation par arrondissement: le cas de quelques quantifieurs prépositionnels
}

\author{
Adler, Silvia, \& Asnes, Maria \\ Université de Haïfa / Université de Bar Ilan \\ sadler@univ.haifa.ac.il /maria.asnes@,gmail.com
}

\section{Introduction}

Dans cet article il sera question d'étudier l'emploi quantificationnel de aux environs de, autour de et aux alentours de, tel qu'il est illustré par la série d'exemples (1) - (4):

(1) Il y avait aux environs del autour de/ aux alentours de 200 bateaux dans le port ${ }^{1}$.

(2) Ça coûte aux environs del autour de/ aux alentours de 3000 Euros.

(3) Inviter aux environs de/ autour de/ aux alentours de 30 personnes.

(4) Ecrire aux environs de/ autour del aux alentours de 25 feuilles d'impression.

Ces expressions, catégorisées par le lexique comme des locutions prépositives, sont communément associées au domaine spatio-temporel. Cette série d'exemples montre pourtant clairement qu'en plus desdits emplois, ces expressions peuvent modifier la quantification fournie par le numéral cardinal, ce qui nous permet de les considérer comme des modifieurs de quantifieurs, tout comme ces autres quantifieurs d"'approximation" que sont les prépositions simples vers ou dans par exemple (cf. Melis 2001 et 2003a; Vaguer 2005 et 2006).

Dans ce qui suit, on enquêtera sur la possibilité d'associer ces expressions au domaine de la quantification. On procédera préliminairement à la question du statut catégoriel de ces opérateurs pour passer ensuite à leurs propriétés en tant que quantifieurs. Comme, en fait, peu de chercheurs ont associé les locutions prépositives au domaine de la quantification (cf. Gross 1977, Melis 2001, 2003a et 2003b, Vaguer 2005 et 2006), la présente étude, qui se situe à la lignée de Melis 2003a, 2003b et constitue une suite à notre recherche antérieure portant sur la quantification prépositionnelle (cf. Adler et Asnes à paraître), se propose essentiellement de mettre en valeur la quantification par aux environs de, autour de et aux alentours de - des opérateurs d'arrondissement non monotones et non bornés - à la lumière de deux autres classes de quantifieurs prépositionnels d'approximation. La première, comportant jusqu'à et $a u$ delà de, qui sont des quantifieurs monotones bornés; la seconde, comportant près de, qui est un opérateur d'approximation asymptotique. Nous partageons avec Melis 2003a et 2003b l'idée essentielle selon laquelle certaines locutions prépositives telles que autour de/aux environs delaux alentours de peuvent fonctionner comme quantifieurs et former non seulement des groupes prépositionnels, mais aussi des groupes nominaux (à l'exemple de près de, jusqu'à, etc.). Pourtant, nous considérons que la quantification en autour de/aux environs delaux alentours de et celle en près de ne relèvent pas du même type : il s'agit d'une approximation par arrondissement dans le premier cas, et d'une approximation par rapprochement asymptotique ${ }^{2}$ dans le second. Selon Melis (2003a), les deux appartiennent à la même classe d'"indicateurs d'approximation purs".

\section{Statut catégoriel}

Il est possible d'établir des équivalences entre aux environs de, autour de, aux alentours de et les modifieurs adverbiaux environ, plus ou moins ${ }^{3}$. Les séquences environ $n$ / plus ou moins $n$ / aux environs de $n$ / autour de $n$ / aux alentours de $n$ (où $n$ représente un numéral cardinal) illustrent une modification du numéral cardinal ${ }^{4}$. Cette mise en relation des modifieurs prépositionnels avec les cardinaux modifiés classiques de type adverbial permet dans un premier temps d'intégrer nos opérateurs dans le domaine de la quantification. 
Ceci dit, la catégorisation syntaxique de nos opérateurs ne va pas sans poser des problèmes. Prenons, par exemple, le critère de la mobilité ${ }^{5}$ mis en jeu pour consolider le statut adverbial de environ et plus ou moins:

(5) environ - plus ou moins 50 caisses / 50 caisses environ - plus ou moins ${ }^{6}$

Qu'en est-il de nos opérateurs ? Si on juge par le critère de la mobilité, il est évident qu'ils ne sont pas des adverbes. D'abord, ils ne sont pas mobiles:

(6) aux environs de, autour de, aux alentours de 50 caisses

(7)*50 caisses aux environs (de)/ autour (de)/ aux alentours (de)

Ensuite, ils ne peuvent pas modifier des verbes tensés ou des adjectifs, comme peuvent le faire environ ou plus ou moins:

(8) Le bébé s'est plus ou moins endormi ${ }^{7}$.

(9) Le pneu est plus ou moins neuf.

Les paraphrases utilisant nos opérateurs sont inimaginables.

Si aux environs de, autour de et aux alentours de ne sont pas des adverbes, s'agit-il alors de prépositions? Observons (10)-(13) illustrant l'emploi prépositionnel classique dans des GPrép de temps (11), (12), (13) ou de lieu (10):

(10) Ils se sont donné rendez vous aux alentours de/ aux environs del autour de la gare.

(11) L'accident a dî se produire aux environs de 17 heures.

(12) Départ autour de 10:00 h.

(13) Tout est rentré dans l'ordre aux alentours de $10 \mathrm{~h} 40$.

Comparons la série d'exemples (10) - (13) à celle de (14)-(19) ci-dessous:

(14) Aux environs de $50 \%$ des billets aériens sont électroniques.

(15) Consommer aux environs de $25 \mathrm{gr}$ de fibres par jour.

(16) Le taxi doit coûter autour de 50 euros.

(17) Cet auteur a écrit autour de 10 livres.

(18) Elle devrait avoir aux alentours de 50 ans.

(19) Chaque train peut contenir aux alentours de 400 voyageurs.

Deux tests montreront que les opérateurs des exemples (14)-(19) ne se comportent pas comme de vraies prépositions, contrairement à la situation illustrée dans (10)-(13):

a. La possibilité d'omission de aux environs de, autour de et aux alentours de pour les exemples (14) (19), mais non pour (10) - (13). Considérons :

(14') $50 \%$ des billets aériens sont électroniques.

(15') Consommer $25 \mathrm{gr}$ de fibres par jour.

(16') Le taxi doit cô̂ter 50 euros.

(17') L'auteur a écrit 10 livres.

(18') Elle devrait avoir 50 ans.

(19') Chaque train peut contenir aux alentours de 400 voyageurs.

Versus 
$\left(10^{\prime}\right) *$ Ils se sont donné rendez vous la gare.

$\left(11^{\prime}\right){ }^{*} L^{\prime}$ accident a dî se produire 17 heures.

(12') *Départ 10:00 h.

(13') *Tout est rentré dans l'ordre $10 \mathrm{~h} 40$.

On peut encore ajouter la propriété typique de dépendance selon laquelle la préposition, en tant que catégorie lexicale, subordonne son complément à une tête externe (par exemple un verbe). Or, nos opérateurs apparaissent en position sujet ou objet direct où ils n'entretiennent pas de dépendance avec une tête externe ${ }^{8}$. Cette propriété va de pair avec la possibilité d'omission de la préposition.

b. La possibilité des questions en combien pour la série (14) - (19). Cet adverbe porte sur le groupe quantificationnel [Modifieur + Num] en laissant intact le complément nominal:

(14"') Combien de billets aériens sont-ils électroniques?

(15") Combien de gr de fibres faut-il consommer par jour?

(17') Combien de livres cet auteur a-t-il écrit?

Dans (10)-(13), la substitution ne se fait pas par un adverbe de quantité, mais par les adverbes sémantiquement appropriés de temps ou de lieu.

(10") Où se sont-ils donné rendez-vous?

(11") Quand l'accident s'est-il produit?

Ce qui a donc la même structure de surface, peut correspondre à deux analyses différentes:

(20) L'accident a dî se produire [GPrép aux environs de [GN 17 heures]]

(21) Cette somme lui permettra d'acheter [GN [GQ aux environs de 10] appartements]

En (20), la préposition aux environs de a dans sa portée le complément 17 heures, et sa suppression est interdite: la préposition est le seul moyen pour établir une relation entre le prédicat verbal se produire et le GN 17 heures. En revanche, aux environs de en (21) n'entretient aucun rapport avec acheter, mais il porte exclusivement sur le numéral 10.

La portée de aux environs de est donc différente dans ces deux exemples : en (20) le GPrép entier sert à modifier le prédicat verbal, en (21) aux environs de, tout seul, modifie la quantité exprimée par 10. Ainsi, en (21) aux environs de 10 appartements est l'objet direct de acheter, contrairement à aux environs de 17 heures, qui est un complément indirect de temps.

Ce qui se passe donc dans les emplois quantificationnels avec aux environs de, autour de et aux alentours $d e$, c'est qu'un item appartenant à la catégorie lexicale de préposition perd la fonction essentielle de cette catégorie, qui est celle d'être un mot relationnel, et acquiert une nouvelle fonction ${ }^{9}$. Aux environs de, autour de et aux alentours de forment ensemble avec le numéral cardinal un groupe quantificationnel, dans lequel ils fonctionnent comme modifieurs de quantifieurs. En fait, Melis (2003a :19) propose pour ce type d'emploi de la préposition de distinguer comportement syntagmatique et comportement paradigmatique: du point de vue syntagmatique, le groupe quantifié présente certaines similarités avec des GPréps (« restrictions sur les combinaisons d'une préposition et d'un groupe nominal » (ibid.)), tandis que du point de vue paradigmatique, le groupe quantifié fonctionne comme un $\mathrm{GN}^{10}$.

Avant de passer à la section suivante, considérons ces autres exemples ((22) - (31)) qui, à première vue, ressemblent aux séquences déjà considérées ci-dessus et qui comportent eux aussi des valeurs numérales, mais qui s'avèrent pourtant très différents en ce sens que leur préposition est non-omissible. Ces cas ne sont pas du domaine de la quantification : 


\section{aux environs de}

(22) La production se situe aux environs de $50 \mathrm{~kg}$ par jour.

(23) La bouteille sera vendue cette année aux environs de 10 - 12 dollars.

autour de

(24) L'Institut de l'entreprise évalue autour de 50 milliards d'euros les programmes Sarkozy et Royal.

(25) La production annuelle d'ylang-ylang oscille autour de 50 à 70 tonnes.

(26) Notre niveau normatif de marge nette se situe autour de $10 \%$.

(27) Sans donner de décompte précis, l'Elysée assurait lundi soir que la totalité des contrats tournait autour de 10 milliards d'euros.

(28) Pour assurer un peu de régularité à ses revenus, qu'elle estime autour de $10000 \$$ par année, elle exige d'être payée au mois.

\section{aux alentours de}

(29) Les frais d'inscription, qui comprennent les boissons, s'élèveront aux alentours de 50 euros.

(30) Les températures maximales devraient se situer aux alentours de $10 / 12^{\circ} \mathrm{C}$.

(31) Le chômage remplace le plein-emploi et se maintient aux alentours de $10 \%$.

Les exemples (22) - (31) contiennent des GPrép de mesure. Melis (2003a : 8-9) cite des exemples de ce genre en parlant de la dénotation approximative de autour de et constate que cet emploi, dans lequel autour de n'est pas déterminant de nom (ou modifieur de quantification), est le plus fréquent si l'on juge d'après Frantext. Cependant, nos exemples (14) - (19) ainsi que les exemples de Melis (Cela coûte autour de 200 euros; J'en ai envoyé autour de 300) prouvent très nettement que autour de et ses synonymes aux environs de et aux alentours de peuvent fonctionner aussi comme des modifieurs de quantification. Autour de peut donc être un quantifieur approximatif aussi bien qu'une préposition approximative.

\section{Sémantique de aux environs de/ autour de/ aux alentours de}

Cette section se propose de fournir une analyse sémantique des quantifieurs prépositionnels aux environs de, autour de et aux alentours de. Il sera montré que ces quantifieurs, approximatifs et non-monotones, fonctionnent comme des opérateurs d'arrondissement.

\subsection{Le concept d'imprécis}

Informellement parlant, nos opérateurs rendent la quantité dans leur portée imprécise ou vague. La notion d'imprécis est à cheval sur deux domaines: celui de la logique floue (cf. Glöckner et Knoll 2001), d'une part, et celui de l'approximation ou de l'estimation, d'autre part.

La logique floue (en anglais fuzzy logic), ayant affaire aux conditions de vérité des propositions contenant des termes imprécis et utilisée en intelligence artificielle, s'appuie sur la théorie mathématique des ensembles flous. Il s'agit essentiellement de la prise en compte d'ensembles définis de façon imprécise, d'un raisonnement approximatif et de degrés de vérité. Autrement dit, étant donné que la logique floue est liée à la subjectivité, la vérification d'une condition est soumise à des degrés allant du 0.0 (faux) à 1.0 (vrai), de sorte qu'il est possible pour Y d'être membre de l'ensemble $\mathrm{Z}$ à un degré de $\mathrm{X} \%$.

Dans le cas par exemple de autour de, aux environs de et aux alentours de 50 personnes, la valeur 50 représente le point de référence, mais au dessous ainsi qu'au dessus de cette valeur la condition n'est pas à $0 \%$ de vérité: le degré de vérification est alors plus faible mais non nul. Par contre, dans le cas de 
exactement 50, qui représente une situation binaire (vrai ou faux), toute déviation de la valeur indiquée (50) résultera en $0 \%$ de vérité.

Résumons: le vague ou le flou repose sur l'impossibilité de fixer l'application référentielle d'un terme. Autrement dit, on ne peut pas affirmer avec certitude si un certain nombre est inclus ou exclu de l'extension de l'expression. Une proposition contenant un terme flou/vague est donc « vériconditionnellement indécidable » (cf. Lupu $2003: 296){ }^{11}$

La notion d'approximation, empruntée elle aussi au domaine des mathématiques, est une représentation inexacte d'une valeur connue ou inconnue. Cette représentation est conçue comme suffisamment proche de la valeur en question. Selon Benett (1987), la méthode approximative est utilisée quand on a des connaissances "qualitatives" sur un certain domaine, c'est-à-dire des connaissances qui ne sont pas quantitativement précises, mais qui permettent des inférences quant au domaine de la dénotation d'un nombre. On a recours alors à un raisonnement mixte, à la fois quantitatif et qualitatif.

En pragmatique, selon la théorie de la pertinence (Sperber et Wilson 1989), l'approximation a affaire à un engagement du locuteur non pas par rapport à la vérité de la proposition mais uniquement par rapport à certaines implications liées à celle-ci. L'approximation est donc liée à l'usage imprécis des concepts précis $^{12}$.

Remarquons que la théorie de la pertinence parle d'une approximation implicite qui ne fait pas partie de la dénotation sémantique de l'énoncé mais qui est contextuellement reconstruite au niveau pragmatique. Quand quelqu'un dit avoir payé 1000 Euros, l'interlocuteur peut prendre cet énoncé littéralement s'il n'interprète que le contenu sémantique ou bien, dans certains contextes, il peut comprendre que 1000 Euros n'est qu'une approximation intégrant les nombres voisins à 1000 (cf. Spector 2006).

Dans les constructions qui font l'objet de notre étude, il s'agit en revanche d'une approximation dénotée explicitement par les modifieurs prépositionnels de quantité. Plus précisément, l'approximation établie par nos opérateurs consiste en un arrondissement d'une valeur numérique ${ }^{13}$.

\subsection{L'arrondissement}

En mathématiques, l'arrondissement est un procédé de remplacement d'un nombre par sa valeur approchée. L'arrondissement peut être par défaut (vers le bas) ou par excès (vers le haut).

Quel type d'arrondissement est-il effectué par nos opérateurs? Autour de, aux environs de et aux alentours de opèrent un arrondissement à la fois par défaut et par excès, à la différence de près de qui fait uniquement un arrondissement par excès (près de est asymptotique ${ }^{14}$ en ce sens que le nombre dans sa portée ne peut jamais être atteint). Considérons :

(32) autour de $10=\{8,9\}$ arrondissement par excès $\{11,12\}$ arrondissement par défaut ${ }^{15}$

(33) près de $10=\{8,9\}$ arrondissement par excès

Le domaine des nombres inclus dans la dénotation dépend des incréments (ou des unités) d'arrondissement. Ainsi, les incréments d'arrondissement croissent avec la croissance de la valeur numérique servant de point de référence.

(34) 3.5 sera arrondi à 3 ou 4

23 sera arrondi à 20

138 sera arrondi à 140

1050 sera arrondi à 1000 ou 1100

28356 sera arrondi à 28000 ou 28500 
Il s'ensuit qu'une séquence telle que autour de 23 peut paraître bizarre dans certains cas, tandis que autour de 23000 est parfaitement acceptable.

Remarquons encore que les incréments d'arrondissement varient selon qu'il s'agit d'une valeur connue ou estimée : dans le cas où la valeur exacte n'est pas connue par le locuteur, l'arrondi peut être plus éloigné du nombre exact que lorsque la valeur est connue.

Nous interprétons les expressions contentant les opérateurs autour de, aux environs de et aux alentours de comme suit:

Dans (3)

(3) Inviter aux environs de/ autour de/ aux alentours de 30 personnes

on considère que le nombre qui se trouve dans la portée du modifieur de quantification est un point de référence - un pivot - par rapport auquel est opéré l'arrondissement. La dénotation de aux environs del autour del aux alentours de 30 inclut des nombres voisins, inférieurs et supérieurs au point de référence. Le domaine de ces nombres n'est borné de façon précise ni à gauche ni à droite. Pourtant, des bornes floues, vagues, y sont impliquées. Celles-ci sont conditionnées par la valeur numérique du point de référence : plus élevée est la valeur numérique du point de référence, plus éloignées sont les bornes présupposées par rapport au point de référence.

\subsection{Monotonicité}

Dans le domaine de la quantification, on distingue des quantifieurs monotones et non-monotones (cf. Barwise et Cooper 1981, Corblin 2002, Kamp et Reyle 1993, Keenan et Stavi 1986). Il existe des quantifieurs monotones croissants (au moins) et d'autres, monotones décroissants (au plus). En ce qui concerne la monotonicité croissante, l'interprétation des GN est telle qu'à chaque fois qu'il y a un ensemble qui appartient à la dénotation de ces GN, tous les ensembles surordonnés y appartiennent aussi (Gamut 1991, 232) :

$$
\begin{aligned}
& \text { a. } A \subset B \\
& \text { b. } A, \subset B
\end{aligned}
$$

où $A \subset A$ ' et si (a) est vrai, alors (b) est aussi vrai, comme dans l'exemple (35) :

\section{(35) a. Au moins deux linguistes (A) sont français $(B)$}

b. Au moins deux chercheurs (A') sont français $(B)$

Pour ce qui est de la monotonicité décroissante, l'interprétation des GN est telle qu'à chaque fois qu'il y a un ensemble qui appartient à la dénotation de ces GN, tous leurs sous-ensembles y appartiennent aussi. La monotonicité décroissante est une sorte d'image miroir de la monotonicité croissante (Gamut 1991, 234).

$$
\begin{aligned}
& \text { a. } A \subset B \\
& \text { b. } A^{\prime} \subset B
\end{aligned}
$$

où $A^{\prime} \subset A$ et si (a) est vrai, alors (b) est aussi vrai, comme dans l'exemple (36) :

\section{(36) a. Deux chercheurs (A) au plus sont français (B) \\ b. Deux linguistes (A') au plus sont français (B)}

D'après Dobrovie-Sorin \& Beyssade (2004:106), dans les GNs construits avec un cardinal modifié, l'expression modifiant le nombre $n$ (représenté par le cardinal) construit une échelle de valeurs et sert également à exclure certains termes de cette échelle. Par exemple, dans le cas des adverbes du type de au moins ou au plus, il y a une échelle de nombres d'ordre décroissant ou croissant respectivement. Ainsi, toujours d'après Dobrovie-Sorin \& Beyssade, au moins dans au moins $n$ enlève tous les nombres inférieurs à $n$ sur une échelle, au plus dans au plus $n$ élimine tous les nombres supérieurs à $n$ sur cette échelle.

A part les quantifieurs monotones (croissants et décroissants), il existe des quantifieurs non monotones, c'est-à-dire qui ne sont ni monotones croissants ni monotones décroissants: 
(37) a. Deux linguistes exactement sont français.

b. Deux chercheurs exactement sont français.

Dans l'exemple (37), les deux phrases n'ont pas les mêmes conditions de vérité en ce sens qu'aucune des deux n'implique l'autre. Le fait que (37b) ne se déduit pas de (37a) prouve que exactement n'est pas monotone croissant. Inversement, l'impossibilité de déduire (37a) à partir de $(37 b)$ montre que ce modifieur n'est pas monotone décroissant non plus.

Pour revenir à nos opérateurs, ceux-ci présentent des ressemblances fortes avec exactement quant à la propriété de monotonicité, ce qui n'est pas surprenant étant donné que dans les deux cas il s'agit de l'établissement d'une identité, forte dans le cas de exactement, faible dans le cas de autour de, aux environs de et aux alentours de.

Voici pour illustrer (38) - (41) :

\section{Monotonicité croissante par rapport à $\mathbf{A}$ :}

(38) a. Autour de cinq linguistes (A) sont français (B)

b. Autour de cinq chercheurs (A) sont français (B)

Le fait qu'il y a autour de cinq linguistes qui sont français ne signifie pas qu'il y a autour de cinq chercheurs qui sont français. On peut avoir plus de chercheurs qui sont français.

\section{Monotonicité croissante par rapport à $\mathbf{B}$ :}

(39) a. Autour de cinq linguistes (A) sont français (B)

b. Autour de cinq linguistes (A) sont européens (B)

Le fait qu'il y a autour de cinq linguistes qui sont français ne signifie pas qu'il y a autour de cinq linguistes qui sont européens. Il est possible qu'il y ait plus de linguistes européens.

\section{Monotonicité décroissante par rapport à $\mathbf{A}$ :}

(40) a. Autour de cinq chercheurs (A) sont français (B)

b. Autour de cinq linguistes (A) sont français (B)

Le fait qu'il y a cinq chercheurs français ne doit pas forcement coïncider avec une quantité égale de linguistes français: il se peut qu'il y ait moins de linguistes français et même une quantité nulle.

\section{Monotonicité décroissante par rapport à $\mathbf{B}$ :}
(41) a. Autour de cinq linguistes (A) sont européens (B)
b. Autour de cinq linguistes (A) sont français (B)

Le fait qu'il y a cinq linguistes européens ne doit pas forcement coïncider avec une quantité égale de linguistes français: il se peut qu'il y ait moins de linguistes français et même une quantité nulle.

\section{Trois types de modifieurs prépositionnels de quantification approximative : étude comparative}

Nous proposons une distinction tripartite de la quantification prépositionnelle approximative $\mathrm{e}^{16}$ : 
1) approximation par atteinte (jusqu'à) ou dépassement (au-delà de) d'une borne

2) approximation par rapprochement asymptotique (près de)

3) approximation par arrondissement (autour de / aux environs de / aux alentours de) $)^{17}$

Dans ce qui suit nous présentons une analyse comparative de ces trois modes de quantification. L'esquisse que nous proposons pour les deux premiers types de quantifieurs servira de point de repère pour une mise en valeur des propriétés particulières des opérateurs du troisième type, telles que celles-ci ont déjà pu être exposées dans la section 3 .

\subsection{Approximation par atteinte ou dépassement d'une borne ${ }^{18}$}

(42) Chaque directeur de recherche peut désigner jusqu'à 3 candidats.

(43) Ce vaste stationnement peut contenir au-delà de 100 véhicules.

Jusqu'á ${ }^{19}$ et au-delà de sont des opérateurs scalaires qui construisent une échelle de nombres de sorte que certains nombres y sont inclus et d'autres en sont exclus. Ainsi jusqu'à dans jusqu'à 3 candidats (42) enlève tous les nombres supérieurs à 3 sur l'échelle construite par cet opérateur. Par contre, au-delà de dans au-delà de 100 véhicules (43) enlève tous les nombres inférieurs et égaux à 100 sur l'échelle construite par cet opérateur. Il s'ensuit que jusqu'à bloque toute quantité supérieure à la valeur donnée et assume de cette manière le rôle d'un superlatif $(\leq)$. Au-delà de fonctionne, lui, comme un quantifieur comparatif de supériorité et marque le dépassement d'une borne inférieure $(>)$. Autrement dit, il bloque toute quantité inférieure ou égale à la valeur donnée. Remarquons que dans le cas de jusqu'à, contrairement à celui de au-delà de, la borne est incluse dans le domaine de la dénotation.

Les deux opérateurs sont monotones. Au-delà de est monotone croissant; jusqu'à monotone décroissant:

(44) a. Au-delà de deux linguistes sont français.

b. Au-delà de deux chercheurs sont français.

(45) a. Jusqu'à deux chercheurs sont français.

\section{b. Jusqu'à deux linguistes sont français.}

Puisque le terme linguiste dans (44a) est un hyponyme de chercheur dans (44b) et que la vérité de (44b) peut être déduite de celle de (44a), au-delà de est un quantifieur monotone croissant.

Puisque le terme chercheur dans (45a) est un hyperonyme de linguiste dans (45b) et que la vérité de (45b) peut être déduite de celle de (45a), jusqu'à est un quantifieur monotone décroissant. ${ }^{20}$

Notre étude se concentre plutôt sur la sémantique de l'approximation, mais il va sans dire que ces opérateurs ont une charge argumentative. Ainsi par exemple dans (46), bien que jusqu'à, de par son sens lexical, impose une limite, cette préposition n'est pas employée pour indiquer une limitation. Bien au contraire, le locuteur communique ici l'idée d'atteinte d'une valeur supérieure à polarité positive.

Malgré les différences sémantiques entre ces deux prépositions, à l'instar de jusqu'à, le but communicatif de au-delà de dans (47) est de souligner le dépassement d'une valeur inférieure (pour une analyse plus détaillée de la valeur argumentative de jusqu'à et au-delà de, cf. Adler et Asnes à paraître).

(46) Il y a jusqu'à dix trains par heure entre l'aéroport de Zurich et la gare centrale.

(47) Ce vaste stationnement contient au-delà de 1000 véhicules.

\subsection{Approximation par rapprochement asymptotique}

Nous entendons par rapprochement asymptotique une situation dans laquelle il y a une tentative de rapprochement vers le nombre donné sans toutefois pouvoir atteindre ce nombre ${ }^{21}$. Ce type d'approximation est illustré par le quantifieur près de: 
(48) Près de 50 nouveaux logements sont subventionnés dans ce quartier.

(49) Notre pays achète près de 100 millions de barils par an.

Tout comme jusqu'à et au-delà de, près de construit une échelle de nombres de sorte que certains nombres y sont inclus et d'autres en sont exclus. Dans le cas de près de il y a une orientation en ce sens qu'il y a une tentative de la part du locuteur à se rapprocher de la valeur exacte supérieure.

Près de dans près de 50 nouveaux logements (48), signifiant "immédiatement inférieur à 50", construit une échelle de nombres de sorte qu'il enlève tous les nombres supérieurs à 50 et tous les nombres qui ne sont pas immédiatement inférieurs à 50 . Le nombre 50 sert de point de référence mais lui-même est exclu du domaine de la dénotation. Près de, qui marque le rapprochement par rapport à une borne supérieure, désigne donc ce qu'on appelle une égalité asymptotique $(\simeq)$.

On reconnaîtra que près de opère aussi une sorte d'arrondissement, tout comme autour de/aux environs delaux alentours de, mais près de en est différent en ce sens qu'il ne fait qu'un arrondissement par excès, tandis que autour de/aux environs de/aux alentours de effectuent un arrondissement à la fois par défaut et par excès.

A l'encontre de jusqu'à et au-delà de, mais tout comme aux alentours de, autour de et aux environs de, près de n'est pas monotone:

\section{(50) a. Près de cinq linguistes sont français \\ b. Près de cinq chercheurs sont français}

(50b) ne peut pas être inféré à partir de (50a) et, inversement, l'énoncé (50b) ne peut pas être inféré de (50a). Il s'ensuit que près de n'est ni monotone croissant ni monotone décroissant. D'une part il peut $\mathrm{y}$ avoir plus de cinq chercheurs français, d'autre part, il se peut qu'il n'y ait pas du tout de linguistes français.

\subsection{Approximation par arrondissement}

Comme déjà suggéré dans la section 3, autour de, aux environs de et aux alentours de sont des opérateurs

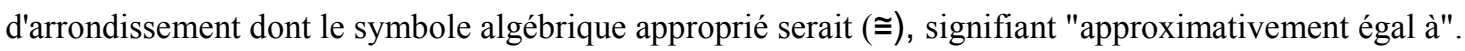
Dans le domaine de la quantification prépositionnelle par arrondissement, le symbole général d'approximation $(\approx)$ sera donc sous-spécifié en $(\cong)$ "approximativement égal à" (autour de, aux environs de et aux alentours de) et $(\simeq)$ "égalité asymptotique" (près de).

Nous avons suggéré dans la section 3.2 que les incréments d'arrondissement croissent proportionnellement à la croissance de la valeur numérique servant de point de référence. Ainsi:

Autour de $3 \rightarrow 2$ ou 4

Autour de $20 \rightarrow\{18,19,21,22\}$

Autour de $100 \rightarrow\{90-99\},\{101-110\}$

Autour de $1000 \rightarrow\{900-999\},\{1001-1100\}$

Ajoutons pourtant à présent que selon Bennet (1987), dans les sciences exactes, l'approximation implique au moins $95 \%$ de précision et $5 \%$ d'erreur maximum.

Précisons encore que dans le processus d'arrondissement, soit le locuteur ne connaît pas le nombre exact, ce qui l'amène à proposer une estimation, soit il connaît le nombre exact mais pour des raisons diverses il choisit de proposer une approximation (cf. Lupu 2003 qui explique cette deuxième possibilité à la lumière de la théorie de la Pertinence).

Le tableau suivant propose une analyse synoptique de la quantification prépositionnelle d'approximation: 


\begin{tabular}{|c|c|c|c|c|}
\hline & Jusqu’à & Au-delà de & Près de & $\begin{array}{l}\text { Autour de laux } \\
\text { environs de/ aux } \\
\text { alentours de }\end{array}$ \\
\hline Symbole & $\leq$ & $>$ & $\simeq$ & $\cong$ \\
\hline Monotonicité & $\begin{array}{l}\text { Monotone } \\
\text { décroissant }\end{array}$ & $\begin{array}{l}\text { Monotone } \\
\text { croissant }\end{array}$ & Non-monotone & Non-monotone \\
\hline Bornage & $\begin{array}{l}\text { Borne } \\
\text { supérieure est } \\
\text { posée }\end{array}$ & $\begin{array}{l}\text { Borne inférieure } \\
\text { est posée } \\
\text { |-------> }\end{array}$ & $\begin{array}{l}\text { Borne supérieure } \\
\text { est posée mais } \\
\text { n'est pas atteinte } \\
\text { borne inférieure } \\
\text { est supposée } \\
\text { exister et elle est } \\
\text { immédiatement } \\
\text { inférieure à la } \\
\text { borne supérieure } \\
\text { (|) -------> | }\end{array}$ & $\begin{array}{l}\text { Pas de borne, ni } \\
\text { inférieure ni } \\
\text { supérieure, mais } \\
\text { celle-ci est quand } \\
\text { même supposée } \\
\text { exister dans les } \\
\text { deux pôles, par } \\
\text { rapport à un pivot } \\
\text { (point de } \\
\text { référence) } \\
(\mid)<---\mid-->(\mid)\end{array}$ \\
\hline $\begin{array}{l}\text { Type } \\
\text { d'approximation }\end{array}$ & $\begin{array}{l}\text { Par atteinte } \\
\text { d'une borne }\end{array}$ & $\begin{array}{l}\text { Par dépassement } \\
\text { d'une borne }\end{array}$ & $\begin{array}{l}\text { Par rapprochement } \\
\text { asymptotique } \\
\text { (arrondissement } \\
\text { par excès) }\end{array}$ & $\begin{array}{l}\text { Par } \\
\text { arrondissement } \\
\text { (par défaut et par } \\
\text { excès) }\end{array}$ \\
\hline
\end{tabular}

\section{Conclusion}

Dans cet article nous avons consolidé la thèse selon laquelle certaines locutions prépositives peuvent fonctionner comme des modifieurs de quantification (cf. Melis 2001, 2003a et 2003b entre autres).

Tout en rejoignant Melis $(2001,2003 \mathrm{a}, 2003 \mathrm{~b})$ en ce qui concerne l'analyse du statut catégoriel de aux environs de, autour de et aux alentours de, notre étude s'est axée essentiellement sur les propriétés sémantiques de ces opérateurs et est allée plus profondément dans le découpage de la notion d'approximation. Nous avons ainsi, pour décrire ces trois opérateurs, introduit la notion d'arrondissement qui permet de préciser davantage leur fonctionnement quantificationnel. La notion d'arrondissement est pour nous un cas particulier de la notion - plus générale - d'approximation. L'arrondissement se distingue des deux autres types de quantification approximative : l'approximation bornée (jusqu'à, au-delà de) et l'approximation asymptotique (près de). Notre étude a révélé que les opérateurs d'arrondissement, tout comme ceux de l'approximation asymptotique et contrairement à ceux de l'approximation bornée, sont non-monotones. Par ailleurs, tandis que aux alentours de/aux environs del autour de opèrent un arrondissement par défaut et par excès, près de opère exclusivement un arrondissement par excès.

Notre étude s'est intéressée essentiellement à l'approximation par arrondissement et s'est servie des autres types d'approximation à titre de comparaison. Il serait bien sûr intéressant dans l'avenir de comparer ce type de quantification aux autres classes proposées par Melis (2003a) : l'approximation par construction d'un intervalle (de $\mathrm{X} \grave{a} \mathrm{Y}$, entre), l'intervalle par définition de l'ordre de grandeur (dans les) et l'approximation par oscillation ( $\mathrm{X} \grave{a} \mathrm{Y}$ ).

\section{Bibliographie}

Adler S., Asnes M., (2004). Les compléments de degré en jusqu'à, Travaux de Linguistique 49, 131-157.

Adler S., Asnes M., (2007). Le monde d'au-delà : une dimension scalaire, Travaux de Linguistique 54, $29-42$. 
Adler S., Asnes M., (à paraître). A la recherche des quantifieurs perdus, Actes du colloque La quantification et ses domaines, 19-21 octobre 2006, Université Marc Bloch, Strasbourg : Presses Universitaires de Caen.

Barwise J., Cooper R. (1981). Generalized quantifiers and natural language, Linguistics and Philosophy 4, 159-219.

Benett S.W. (1987). Approximation in Mathematical Domains, Proceedings of the tenth International Joint. Conference on Artificial Intelligence, Los Altos, CA http://dli.iiit.ac.in/ijcai/IJCAI-87-VOL1/PDF/048.pdf

Borillo A. (1998). L'espace et son expression en français, Paris : Ophrys.

Corblin F. (2002). Représentation du discours et sémantique formelle. Introduction et applications au français, Paris : P.U.F.

Defrise Ch. (1989). Lexical description for NLP: The case of the French adverb Presque in Machine Translation 4 (3), Springer Netherlands, 0922-6567 (Print) 1573-0573 (Online), 195-232.

Dobrovie-Sorin C., Beyssade C. (2004). Définir les indéfinis, Paris : CNRS Editions.

Gamut L.T.F. (1991). Logic, language and meaning. Chicago : University of Chicago Press, Volumes 1 and 2.

Glöckner I., Knoll A. (2001). Fuzzy Quantifiers: A Natural Language Technique for Data Fusion, in Proceedings of the Fourth International Conference on Information Fusion (Fusion 2001).

Gross M. (1977). Grammaire tranformationnelle du français : syntaxe du nom, Paris : Larousse.

Hadermann P., Pierrard M., Van Raemdonck D. (2007). La scalarité : autant de moyens d'expressions, autant d'effet de sens, Travaux de Linguistique 54, 7-15.

Ilinski K. (2003). La préposition et son régime: étude des cas atypiques, Paris : Champion.

Kamp H., Reyle U. (1993). From Discourse to Logic, Dordrecht : Kluwer.

Keenan E. L., Stavi J. (1986). A semantic characterization of natural language determiners, Linguistics and Philosophy, 9, 253-326.

Kleiber G. (1987). Quelques réflexions sur le vague dans les langues naturelles, in Etudes de linguistique générale et de linguistique latine offertes en hommage à Guy Serbat, Paris, Bibliothèque de 1'Information, 157-172.

Krifka M. (1999). At least some determiners aren't determiners, in The semantics/pragmatics interface from different points of view, K. Turner (ed.), Elsevier, 257-291.

Leeman D. (2005). La préposition jusque, in Le mouvement dans la langue et métalangue, P. Dendale (éd), Metz, Université de Metz, collection Recherches Linguistiques 27, 103-119.

Lupu M. (2003). Concepts vagues et catégorisation, Cahiers de Linguistique française 25, 291-304.

McNally L. (1998). Existential Sentences Without Existential Quantification, Linguistics \& Philosophy 31, 353-392.

Melis L. (2001). La préposition est-elle toujours la tête d'un groupe prépositionnel?, Travaux de Linguistique, 42-43, pp.11-22.

Melis L. (2003a). Les quantificateurs approximatifs de type prépositionnel, Verbum 25, 5-24.

Melis L. (2003b). La préposition en français. Editions Ophrys.

Milner, J. C. (1978). De la syntaxe à l'interprétation. Paris: Le Seuil.

Nouwen, R. (2006). Remarks on the Polar Orientation of Almost. in: van de Weijer, J. and Los, B. Linguistics in the Netherlands 23-1, 162-173. John Benjamins Publishing Company.

Spector B. (2006). Aspects de la pragmatique des opérateurs logiques. Thèse de doctorat. Université Paris VII- Denis Diderot (sous la direction de A. Rouveret).

Sperber D., Wilson D. (1989). La pertinence. Communication et cognition, Paris : Minuit.

Vaguer C. (2005). Dans les + numéral : un déterminant de quantification faible?, Travaux de Linguistique 50, 113129.

Vaguer C. (2006). Approche du sens des prépositions: le cas de vers, Modèles linguistiques 54, tome XXVII-2, 3750. 
1 Beaucoup de nos exemples sont tirés du moteur de recherche Google.

2 Pour la définition du rapprochement asymptotique, voir section 4.2.

3 On peut ajouter également grosso modo et en gros. Ces termes présentent avec plus ou moins des propriétés distributionnelles différentes de celles de environ, lequel ne peut pas servir de connecteur en position initiale:

(a) Il est venu environ/plus ou moins/grosso modo /en gros 10 personnes.

(b) Grosso modo/en gros/*environ/plus ou moins, il est venu 10 personnes.

(c) Il est venu 10 personnes grosso modo / en gros/ environ/ plus ou moins.

4 Cf. Krifka (1999).

5 McNally (1998).

6 Mais: presque 50 caisses - * 50 caisses presque.

7 Les paraphrases avec environ ne sont pas possibles étant donné que environ sélectionne un terme à valeur exclusivement numérique. Par contre, des termes qui pourraient être considérés comme synonymes (presque ou à peu près) ne posent pas de problème.

8 Melis (2003b : 34-35) ajoute encore d'autres cas de fonctionnement atypique des prépositions qui ne sont pas subordonnées à une tête externe. Cf. également Ilinski (2003) pour les emplois atypiques de certaines prépositions.

9 Pour une discussion élaborée du statut catégoriel des quantificateurs prépositionnels d'approximation, voir Melis (2001, 2003a, 2003b).

10 On se rappellera également des tests proposés par Milner (1978) permettant de distinguer les vrais GPréps des ceux qui y ressemblent mais qui ne le sont pas. Par exemple, on ne peut extraire un GPrép d'un autre GPrép qui le contient: *c'est [GPrépde Zola] que je me suis intéressé [GPrépaux livres] (Milner 1978 : 56). Par contre, on admet l'extraction d'un GPrép contenu dans un GN quantifié : c'est [GPrépde Zola] que j'ai lu [GN deux livres/beaucoup de livres/autour de cinq livres]. Ceci pourrait consolider davantage le fait que les groupes quantifiés en autour de laux alentours de laux environs de fonctionnent comme des GNs.

11 Voir aussi Zhang (1998: 15) et Kleiber (1987: 161-162).

12 Cf. Lupu (2003) pour le point de vue de la théorie de la Pertinence en ce qui concerne l'approximation.

13 Il serait bien sûr intéressant de comparer aux alentours de/autour de/aux environs de trente avec une trentaine où la notion d'approximation est lexicalisée à l'aide du suffixe-aine.

14 Cf. Defrise (1989) pour le traitement de presque en tant que marqueur asymptotique. Voir aussi Nouwen (2006) pour almost.

15 Autour de 10 dans l'exemple (32) peut être paraphrasé par entre 8 et 12 où entre...et est un modifieur de quantification et où les nombres 8 et 12 représentent les bornes inférieure (8) et supérieure (12).

16 Melis (2003a) propose de distinguer 5 types de quantification approximative :

a) approximation pure : près de, autour de

b) approximation par approche de la limite supérieure : jusqu'à

c) approximation par construction d'un intervalle : de X ̀̀ Y, entre

d) l'intervalle par définition d'un ordre de grandeur : dans les

e) l'approximation par oscillation $: X \grave{a} Y$

Comme les types (c), (d) et (e) ne font pas l'objet de la présente étude, notre classement des types d'approximation est différent et ne coïncide pas nécessairement avec celui de Melis. Ainsi, nous distinguons entre l'approximation en près de et celle en autour de et nous ajoutons l'approximation en au-delà de dont Melis ne fait pas mention.

17 Remarquons que tous nos quantifieurs d'approximation (jusqu'à, au-delà de, aux environs de, autour de, aux alentours de, près de) opèrent, en tant que locutions spatio-temporelles, une localisation externe (cf. Borillo 1998). La non-convergence entre site et cible serait-elle à la base de la notion d'approximation? Le cas de la préposition simple dans, participant à la quantification floue ne semble pas constituer une vraie exception: dans les 10 invitations 
voudrait dire que le locuteur situe la quantité précise quelque part dans les incréments potentiels de l'ensemble dont le pivot est 10 unités.

18 Cf. Adler et Asnes (2004), (2007).

19 Pour jusqu'à, Melis (2003a) parle d'une approximation par approche de la limite supérieure. Il s'agit d'après nous non seulement de l'approche mais également de l'inclusion de la limite supérieure dans la dénotation de jusqu'à (cf. Adler et Asnes à paraître).

20 Il existe un paramètre supplémentaire distinguant ces trois types de quantifieurs, qui ne pourra pas être traité dans les limites étroites de cette étude, et qui consiste dans la possibilité d'intensification des prédicats. Précisons: il s'agit de vérifier pourquoi jusqu'à et au-delà de fonctionnent comme des opérateurs de scalarité (cf. Adler et Asnes 2004, 2007 ; Hadermann, Pierrard et Van Raemdonck 2007; Leeman 2005) permettant un mapping entre une valeur sur une échelle de propriété ou d'événement et une valeur sur une échelle de degré, comme par exemple dans épouvanter jusqu'àl au-delà de l'horreur, ce qui ne semble pas fonctionner avec autour de, aux alentours de, aux environs de et près de.

21 Melis (2003a) considère que près de a la même valeur que l'adverbial à peu près, à savoir les deux signalent que « la quantité visée est proche de celle que le pôle signifie » (Melis 2003a : 7). 